\title{
Data Modeling and Query Optimization Technique in Business Intelligence Applications
}

\author{
Zafar Ali Khan and M. N. A. Khan \\ Shaheed Zulfikar Ali Bhutto Institute of Science and Technology, \\ Islamabad, Pakistan \\ zafar786786@gmail.com,mnak2010@gmail.com
}

\begin{abstract}
The automated systems that support in decision making process are known as Business Intelligence Systems. In the past 10 years, the importance of Business Intelligence Systems has gained widespread acceptance by the industry. There are a large number of organizations that are now dealing with great number customers and enormous amount of data. To meet their growing computation needs and enhance their timely decision making capability, these organizations are highly relying on business intelligence applications to meet the challenges posed by the today's competitive market. To overcome these challenges, the companies and organizations integrate enterprise applications with the real time systems such as decision support systems and business intelligence systems. There are several companies and organizations that are using Business Intelligence tools to get knowledge from the history data and to make plan and educated guesses for the future trends and demands. Many companies know the importance and role of Business Intelligence to make effective and timely decisions in order to enhance their productivity and keep abreast with the new market demands.
\end{abstract}

Keywords: Business Intelligence, Data Modeling, Query Optimization, Materialized Views, Data Warehouse

\section{Introduction}

Business Intelligence is used in the organizations to analyze the data for making decisions. Resultantly, organizations examine their businesses through implementing BI tools. But there are still some organizations that face challenges to be part of BI and few have implemented BI application but they are unable to get maximum benefits from BI because they are not familiar with its concepts and are not fully ready for BI. Enterprise managers take decisions on the basis of data provided by BI tools so nowadays no one can ignore the importance of BI and BI tools. Business intelligence technologies provide decision support system.

A new hybrid system is proposed in this study which will be built by getting data across different sources and then combined and organized in a centralized database. After that transformation process will be applied to it to refine and make it a base for reporting system. The different models have been studied and then the information provided by them collected and tried to find out a system which can generate an organized and intelligent support system to assist higher level executives and company owners to take decisions resulting in increased profits and revenues. In this way the proposed model would be used to build another database which collects data from different data sources and deposit into a centralized location. For Future work, a new Decision Support System would be developed with the combination of Database as well as Java Application. Also it would be used to

Received (February 14, 2018), Review Result (May 2, 2018), Accepted (May 7, 2018) 
categorize DSS into individual and group DSS, spreadsheet DSS, solver oriented DSS etc.

\subsection{Tools used for $B I$}

Different types of Business Intelligence tools are available in the market and their categories are given below:

- Enterprise BI tools.

- Database or packaged BI tools.

- Visual Data Discovery tools.

- Reporting tools.

- OLAP tools.

- Innovative or functional tools.

Some of the famous BI tools are listed below:

- SAP BusinessObjects

- IBM Cognos Series 10

- MicroStrategy

- Oracle Enterprise BI Server (OBIEE)

- Oracle Hyperion System

- QlikView

- SAS Enterprise BI Server

\subsection{Business Intelligence Decision Support System}

Nowadays, the environment in which organizations are working is becoming more and more complex. Continuous business pressures, competitor's strategies, and knowledge of current affairs play significant role for building healthy business. Therefore, computerized support has become the major components for every organization to deal with the customers and competitors. Hence, this is the era of DSS, some of the main tools which can apply for all type business environmental factors such as; markets, customer demand, technology, and societal factors for generating best decision from the list of alternatives. You [2] described that a DSS must consider four phases for complete decision making process such as; Intelligence, Design, Choice and Implementation. Organizations own DSS for presenting themselves proactive and anticipative. The development of DSS can be based on data or model. According to Turban, the major classification of DSS is based on Data Oriented DSS and Model Oriented DSS. Although other scholars also categorize DSS into individual and group DSS, spreadsheet DSS, solver oriented DSS etc.

\section{Literature Review}

Ciobaju et al. [1] described BI synchronization on distributed environment and provided solution through network for internet servers. In this paper, the authors propose a common solution for all BI application that runs on different servers on the Internet. For this purpose, the authors performed a case study on Microsoft BizTalk server as a BI system and called this research a PrO-Processor. The authors conducted this research because there was a need of that kind of research through which we can communicate on different servers with the help of Internet where BI applications are installed. The authors provided a common synchronization architecture that is intra coordination among different BI applications installed on different servers. The research caters for configuration of BI application installed on their servers. They also suggest the security of the communication by using TLS and 
SSL protocols. The system also provides facility for tracking records of all clients. The servers maximize the number of client demands and minimize the server hardware requirement. In the future, more values can be added in this by creating the bigger BI solutions such as Oracle, SAP etc. We can also go for the different versions of the same Research. In the research, Pro processor software is introduced. Pro processor is software that is used for as it is clear that the companies need BI system. The Pro processor generates a report that is legal document used by the public institutions. The main module of the Pro Processor are; configuration module that is responsible for configuration of the master data, the data acquisition module, that create link for the Pro processor to communicate with other local system of the host company, the data processing module, that create reports on the requirement gathered, the eSafe that take the responsibility of security issues and finally the report dispatching module that generates the report for public institutions. The architecture of Pro system is also introduced, and it gets the data from the customer into the internal system.

Cvitas [7] explained Business Intelligence importance in enterprises. BI plays a vital role in quick decision making by analyzing data provided by data warehouse. The BI decision making is dependent on the source data. The paper explains alternative way of using strictly structured data. The paper gives an overview of many proposed techniques for information extraction. In the research paper Cvitas explained information extraction and retrieval, information extraction types, machine learning, machine learning methods for information extraction and information extraction in BI. He explains that BI is set of application and technologies that is used for gathering, storing, accessing and analyzing data for decision making. The BI applications are dependent on data warehouse and data warehouse is responsible to provide clean and standard data while the data warehousing is depending on the mature process of ETL to When there is more complex analysis and reports, BI needs import of data. If the data is structured then ETL process is used. But when the data is unstructured then the textual format document is used rather than ETL.

Not all types of information are relevant and can be used in BI. In the paper information extraction from the BI system described in detail. He explained that information extraction can play two different roles depending on the data or text it processes. The first step can give you complete knowledge which can be used for the decision making while in the second role the set of data provided need some more processing to get accurate and complete knowledge. He explained information extraction types, methods and symbolic techniques. He also explained the machine learning issues. According to the paper machine learning methods for the information extraction are already classified examples. According to these learning set, system has to predict for the unseen examples. He also explained unsupervised machine learning because the machine learning disadvantage is that it needs a huge set of learning pattern and that make the entire process very time consuming and complicated. It also takes lots of manual work for the machine learning. Due to these issues unsupervised machine learning is of high importance for the information extraction in business intelligence systems. The paper explained that the real unsupervised machine learning is clustering and it works on the smallest case and the algorithms are design. Clustering is the statistical method and it automatically group data into cluster or groups. And then it replace with similar objects. Expansion is another machine learning method and it belong to the weekly supervised machine learning. In the paper he also explained some other method for machine learning e.g. self- learning, co-learning and active learning. In the end the paper explained how information can be extracted in business intelligence. Analyzing large amount of data for the purpose of decision making and to compete 
the organization in the marketplace. BI depends on the data warehouse and data warehouse is designed by the support of mature ETL process. When all the data is transformed into the structured form in data warehouse then all kind of data mining algorithms can be apply. All the information provided by the BI is not useful for every department. The information varies from department to department and they pick the information according to their need and according to their strategic need. Information extraction from text base or unstructured data is one step in BI. So the idea provides future direction for text base information extracting.

Wang et al. [2] defined the importance of Cloud Computing and Business Intelligence together in this research paper. The authors explain the technologies and architecture of cloud computing and BI. It is also compared with tradition BI and examines the advantage and feature of cloud computing based BI. In the research paper, it explains the complete architecture of BI and describe its components. The paper described the BI architecture and explains its major components.. The whole system is scheduled for meta data management. To compare traditional BI and the cloud-based BI, the authors explained the traditional BI and showed graphically that there are four categories namely Front End Application, Data Storage, Data integrity, Data Resources. They presented the physical architecture of Cloud Computing based BI.

Dayal et al. [6] explained the entire integration flow in the future BI. Authors also explained why it is required and its benefits. With increasing demand of data warehousing ETL design has become more complex so there is a need to design such system having data integration facility. They also explained the next generation data integration flow in detail. Authors described an example scenario that was used throughout the research. The enterprise database is based on the structure data but in future we expect that the enterprise data will have semi-structure and unstructured data for its data warehouse. In the current system, the ETL process is on way, from operation data to the enterprise data warehouse. In future it may support some more functionality. The outcome of data cleaning could be part of the operation system to improve accuracy and reduce cleansing work. The paper also explained the lifecycle describing challenges, design and implementation of optimal flow for the real time BI. The paper gives future directions and explains the drawback of current data warehouse process. In the paper the importance of automated process of data warehousing is described because it will remove the labor work. It also explains that there is need for optimizing the process because in future we need the real time data to be part of the data warehouse.

Al-Aqrabi et al. [4] explained Business Intelligence importance in enterprises. Authors explains that BI plays a vital role in quick decision making by analyzing data provided by data warehouse. The BI decision making is dependent on the source data. The paper explains alternative way of using strictly structured data. The paper gives an overview of many proposed techniques for information extraction.

Wang et al. [5] explained information extraction and retrieval, information extraction types, machine learning, machine learning methods for information extraction and information extraction in BI. Authors explains that BI is set of application and technologies that is used for gathering, storing, accessing and analyzing data for decision making. The BI applications are dependent on data warehouse and data warehouse is responsible to provide clean and standard data while the data warehousing is depending on the mature process of ETL. When there is more complex analysis and reports, BI needs import of data. If the data is structured then ETL process is used.

Sanjay et al. [6] defined the knowledge management from Business Intelligent systems. They explained that there are lot of organizations that implement BI systems for dynamically creating and managing information that enables real time 
response. And that response plays a major role in decision making for enterprises. The study describes that the BI systems are heart of organization and it needs to be optimized. The paper focuses on the importance that the information provided by BI needs to be converted into Knowledge so that the decision can be easily taken by the higher management. The organization often has the process to convert the data into information and then this information helps in decision making but this paper is about the automatically managing of meta data knowledge through BI system. They focus on knowledge base process to simulate business scenarios and performance matrices with critical functions to build metadata knowledge from the data provided by the data warehouse. In this paper the key functionalities of BI tools are explained that helps in decision making for higher management.

Jun [10] described that enterprise executive take decisions on the basis of data provided by the different sources like enterprises. In BI lots of tools have been developed to support the enterprise manager in decision making. But the tools are designed according to the requirements and have some limitation. Some tools developed for small scale business and some are developed for large scale business like telecom subscribers. Some tools have less data analysis capabilities and reporting limitation. But in this paper efficient data mining tools, BI framework and computation intelligence techniques have been introduced for the large scale organizations. The research was done on neural network computing and also used optimizer tool for better results. In this research other tools like predictor tool, classifier tool and optimizer tool have been used. The paper explained the BI components like; OLAP, Data Mining CRM, Database Mining, Decision Support System, Knowledge Management, Geographic Information System and Visualization. Every component is explained in a better way.

Mathrani [8] described the query optimization idea for speedy data retrieval. The authors used indices based on best practices of standard databases. The first thing to be considering for best database performance is that the database design should be correct, and it is the basic and important step towards the best performance of database. The author focused that all tables should be in $3 \mathrm{NF}$. When database design is established in $3 \mathrm{NF}$ then tuning of the logical database structure can be done by the indices techniques. Being an important database object, an index is used to fetch the data speedily. Using indices properly will limit the I/O operation and improve the query response time.

Ren [3] focused on scenario in the sub-queries when the objects and conditions of the outer and inner query are the same - a mechanism called intra query redundancy in sub-queries. It affects the query performance when correlated nested queries exist. The authors described query un-nesting for performance improvement in this situation. But sometime sub-query is needed when aggregate calculation is involved. Reference [9-25] outlined various software engineering and machine learning techniques in different domains.

\section{Proposed Model}

Our proposed model is about data modeling and query optimization in BI systems. To implement this system, the traditional approach of Data Warehouse (DWH) is used. A DWH is simply a consolidation of data from a variety of sources that is designed to support strategic and tactical decision making. Its main purpose is to provide a coherent picture of the business at any point in time. Using various Data Warehousing toolsets, users are able to run online queries and 'mine" their data.

In this proposed model, we have made an attempt to improve the performance of the data extraction in BI Systems, for this purpose the traditional approach is used 
and then added some more components and objects in the process. Hence, the proposed model improves the performance of the system. We have used ETL process which is performed using different tools like Data Stage, Informatica etc., to design DWH and data marts.

The proposed system aims at enhancing efficiency of data extraction in BI reporting. This issue has been observed a lot of time during research in DWH and BI reporting. Many companies face problems of diverse nature due to this as their Key Performance Indicators (KPIs) are severely affected. To achieve their target and KPI, the companies need proper utilization of BI application and DWH. Generic data warehouse architecture is shown in Figure 1.

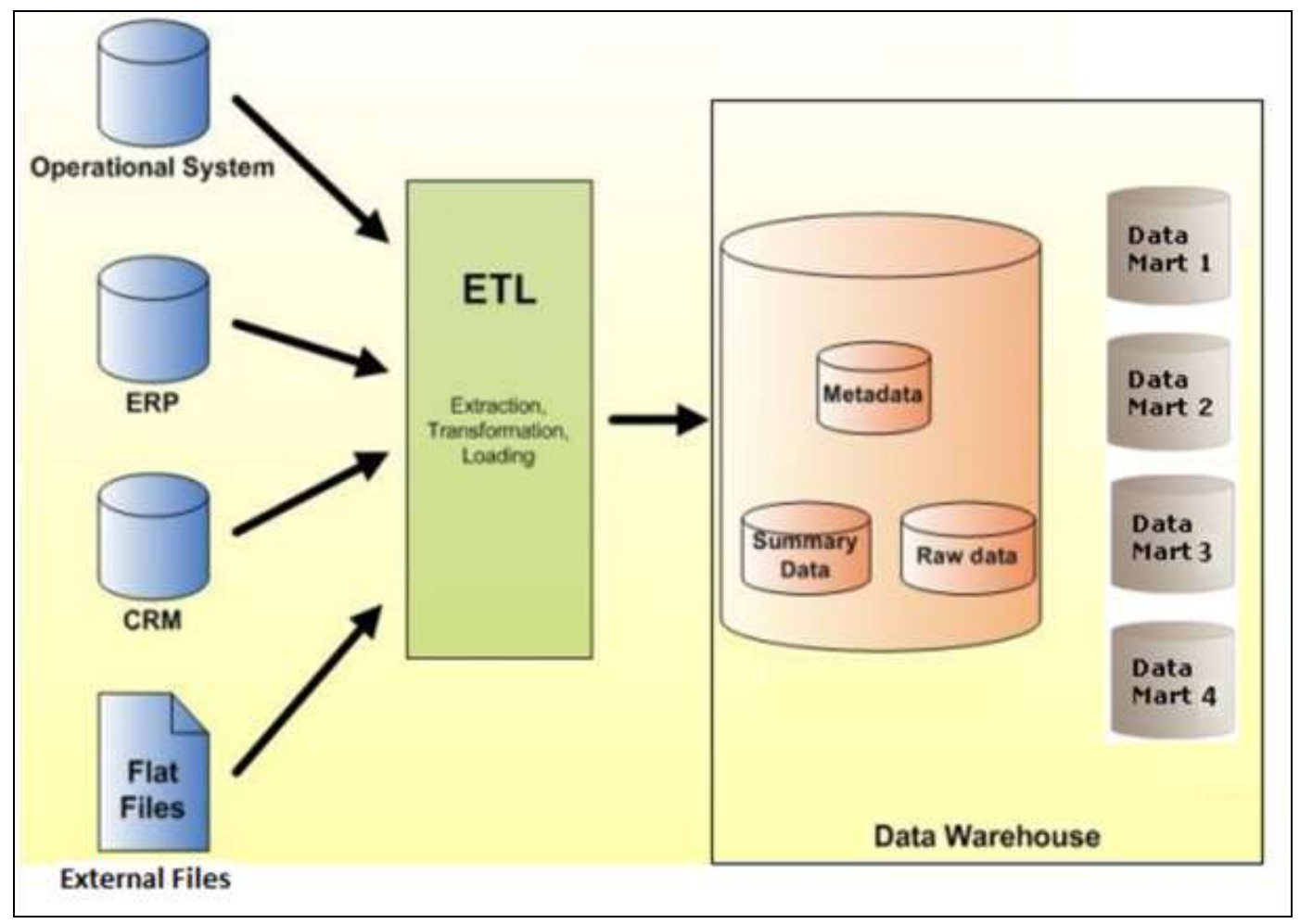

Figure 1. Data Warehouse Architecture

\subsection{Components of the Proposed System}

The key components used in the proposed system are listed below:

1. ETL Process.

2. Data Marts

3. Current Active Dataset

4. Historical Dataset

5. Materialized Views

6. Reporting Tools

7. Ad Hoc Reporting

8. Enterprise Reporting.

To implement this system, ETL process is used to get Data Mart and from the Data Mart different Datasets are created such as Current Active Dataset and Historical Dataset. 


\subsection{Current Active Dataset}

The main aim of this research is to create Current Active Dataset that contain data for the previous year. The retention policy for the data was decided to keep data pertaining to the last one year. Because $90 \%$ of the reporting is performed from the last one year data in order like to compare data trends daily, weekly, monthly and quarterly. So we decided to create Current Active Dataset to perform efficiency of the system to prove that it works well and improves the performance more than 50\%. To get more benefit from the system, materialized views are created on the Current Active Dataset. Materialized views are created during the ETL process of daily batch execution.

\subsection{Historical Dataset}

Historical dataset works like archives and all the historical data is stored here. This dataset was normally used once in a year. With the support of the DBA, it can be used annually and we can easily create the required reports. For this DBA can easily stop all unnecessary processing and ensure the availability of the session or processing for the reporting. A pictorial representation of data warehouse with active and historical dataset is shown in Figure 2.

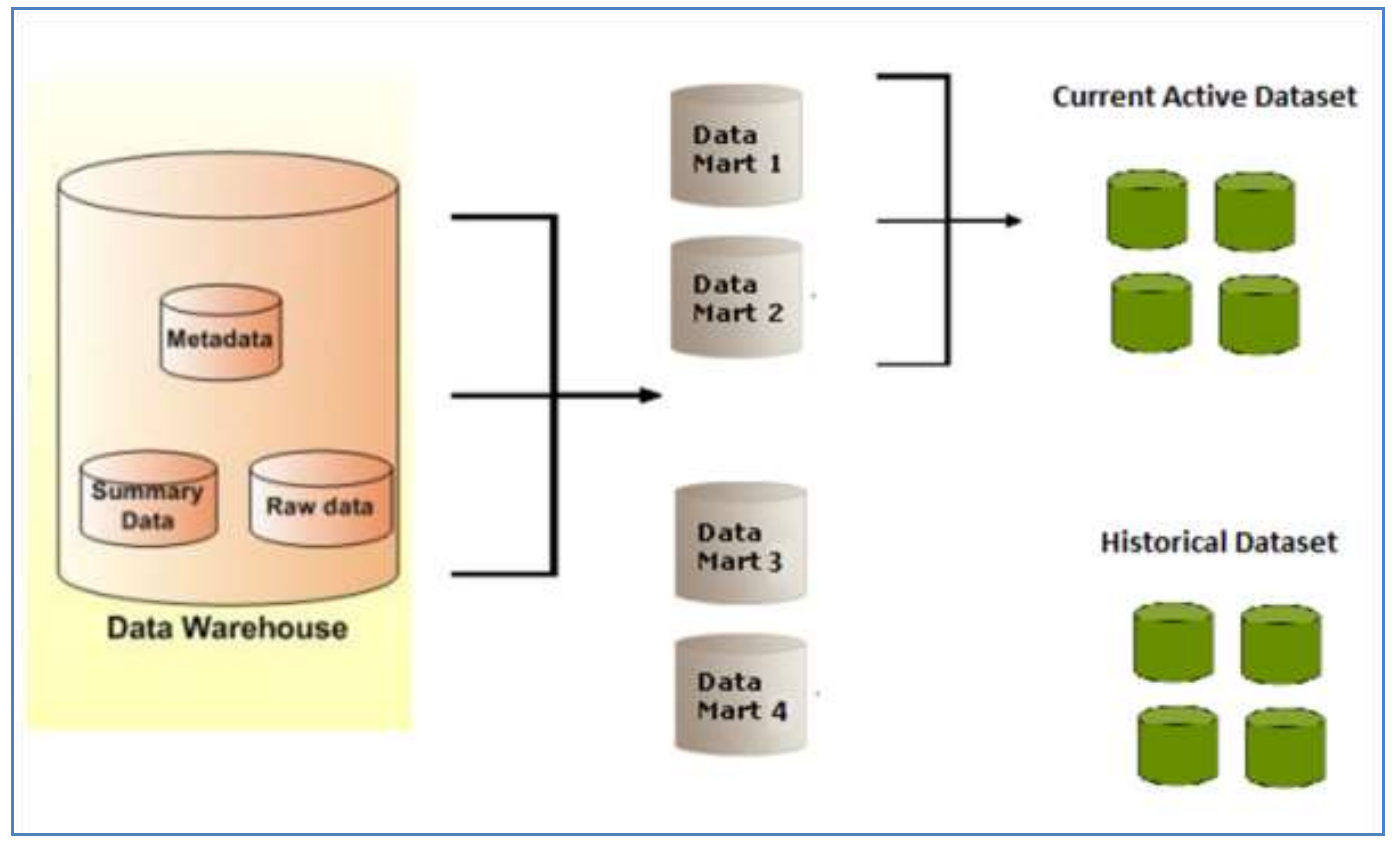

Figure 2. Data Warehouse with Active and Historical Dataset

\subsection{Materialized Views (Partitioned)}

Materialized views have the ability to speed up queries while being transparent for the queries in a similar way as indexes of the partitioned tables are. That means that we do not have to modify our queries in order to benefit from these structures. Unlike an ordinary view which is only a stored select statement that runs if we use the view, a materialized view stores the result set of the select statement as a container table. Here we discuss how the materialized views to improve the performance of system. The architecture of BI solution with dataset and materialized view is shown in Figure 3. 


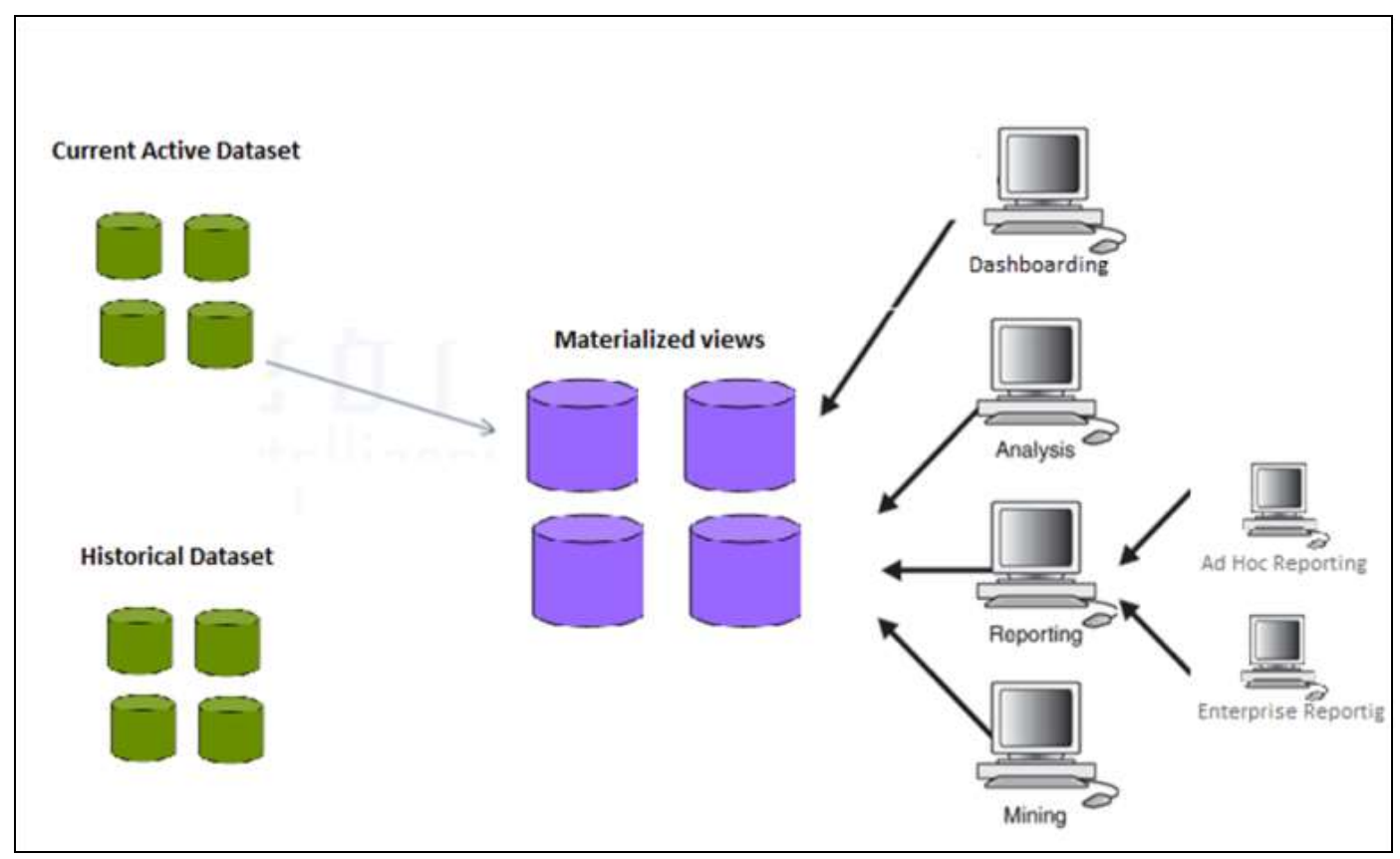

Figure 3. BI Solution with Dataset and Partitioned Materialized Views

For the implementation of the proposed system, we used IBM Cognos. However, other tools like SAP Business Object, Qlik Sense and Qlik View can also be used.

\section{Implementation - Experimentation Sample Query}

To check the efficiency of the system, a complex query was performed from the practical environment. From the last 6 years I am working in Telecom with the similar kind of environment and we are facing issues with reports execution performance. I choose the same kind of environment for my model implementation/ experimentation. I used IBM Cognos as reporting tool and Oracle 11.0.2.4 as data warehouse solution.

The query's complex condition and multiple case statements were used so that the performance could be checked before and after the implementation. When the query was tested before the implementation of the system it took two to three hours for execution. The same query was then performed in Cognos using embed query option, but it increased the performance with extracting the report using the performance of the other reports instead. When the system was implemented and the same query was used to get the result it was executed within 5-10 minutes. Similarly, it also improved the performance in Cognos.

\subsection{Experimentation and Results}

For the past few years I am working in Telecom and I will validate my model on Telecommunication data. To test the model I created two different schema, one I have used for the normal query execution and testing and then using the embedded query option in Cognos to check the performance of the report. The other I have used on the model which I have built and the query in that environment and used the portioning and materialized views. We used Oracle 11.0.2.4 as our backend database and used SQL Developer. To examine the overall performance of our model, we developed several queries and executed them on the classic as well as on our model. Performance has been measured through the output and execution time. The detail description of these queries is described as follows: 


\subsubsection{Sample Query 1}

Since performance is measured in terms of statistics, so to reset the session level statistics connect as anew session/connection and check the statistics with the query shown in Figure 4 and 5 below:

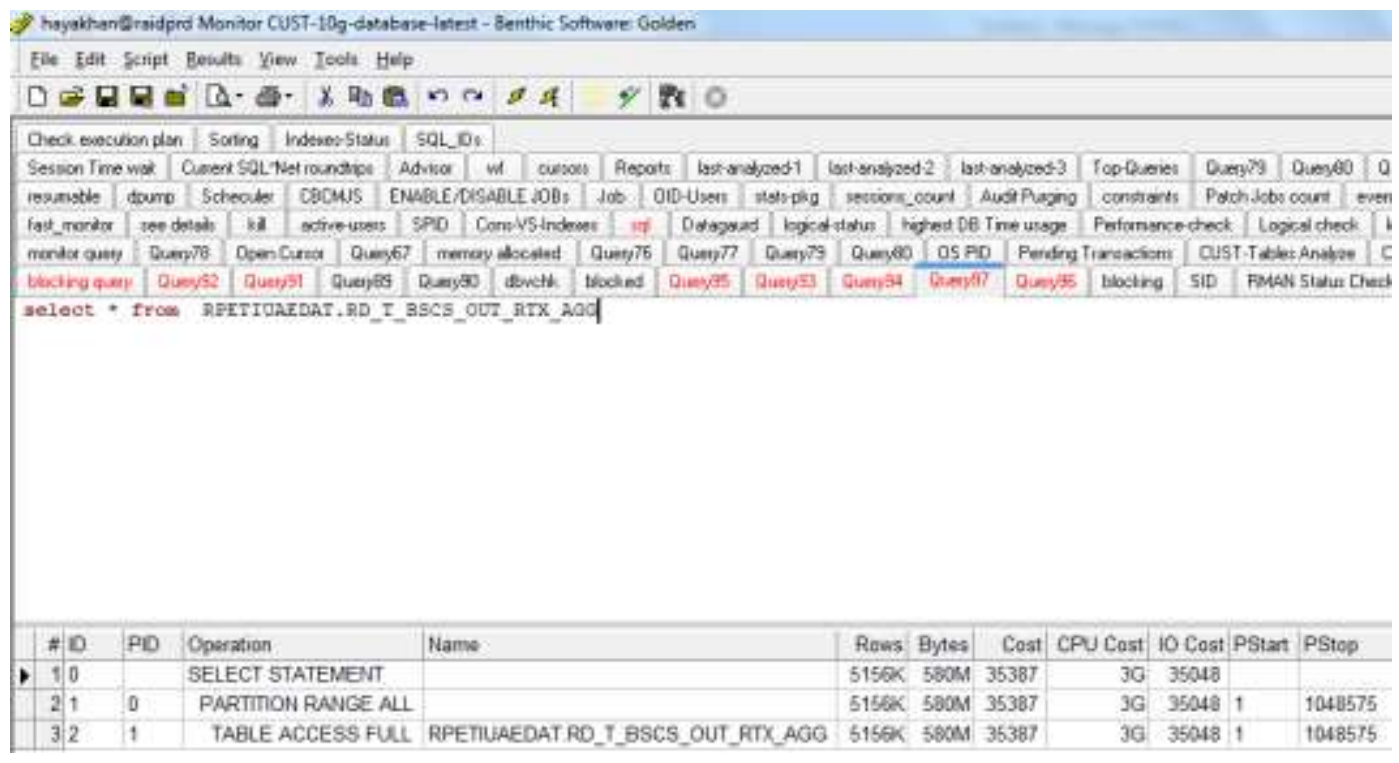

Figure 4. Query from the source table.

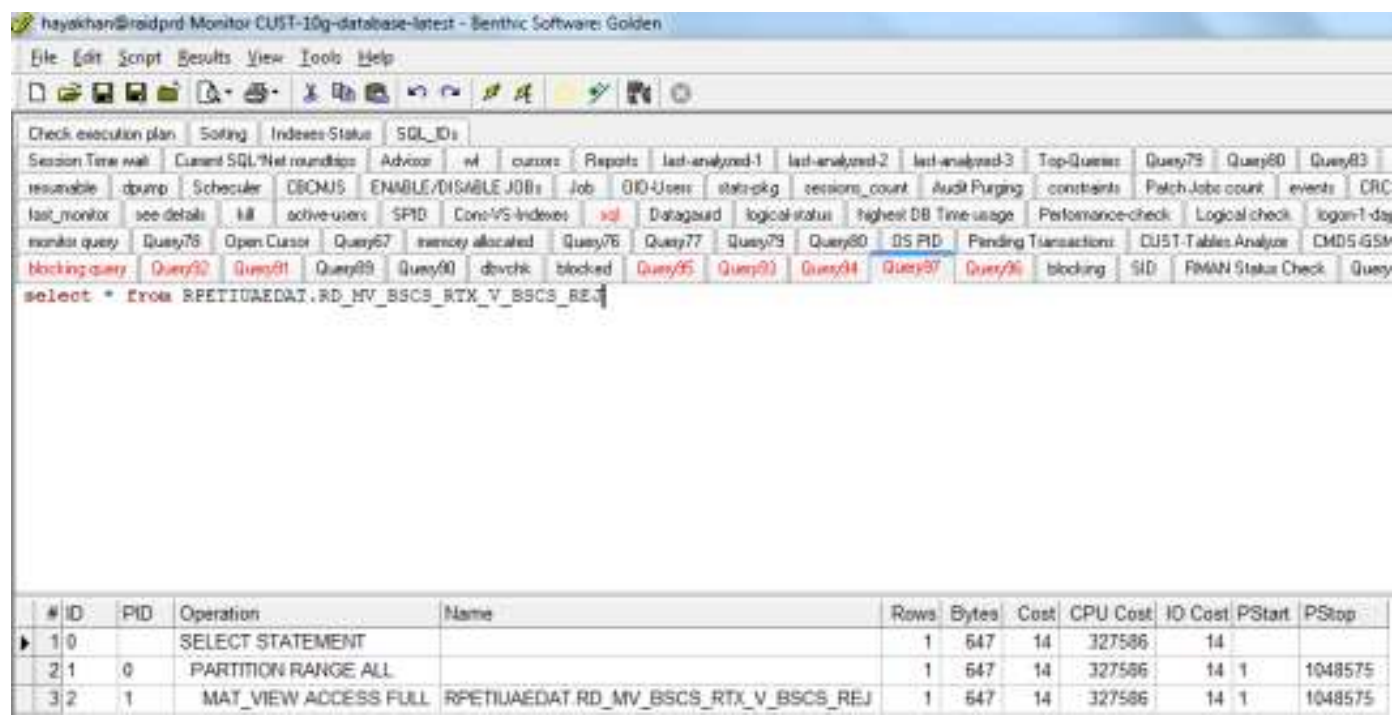

Figure 5. Query from the MV

Table 1. Comparison table between Reports Fetched from DWH and MV

\begin{tabular}{|l|l|l|}
\hline $\begin{array}{l}\text { No of Records } \\
\text { in the Report }\end{array}$ & $\begin{array}{l}\text { Time to } \\
\text { Execute }\end{array}$ & Source \\
\hline 2963 & 1184 & DWH \\
\hline 2963 & 13 & $\begin{array}{l}\text { Materialized } \\
\text { View }\end{array}$ \\
\hline
\end{tabular}




\section{Discussion}

Business Intelligence is used in the organizations to analyze the data for making decisions. Resultantly, organizations examine their businesses through implementing BI tools. But there are still some organizations that face challenges to be part of BI and few have implemented BI application but they are unable to get maximum benefits from BI because they are not familiar with its concepts and are not fully ready for BI. Enterprise managers take decisions on the basis of data provided by $\mathrm{BI}$ tools so nowadays no one can ignore the importance of BI and BI tools. Business intelligence technologies provide decision support system.

In traditional applications, the paging query processes at the database server, starting from fetching data, satisfying query condition at a time and then the resultant row-set is delivered to the client cache showing page wise results. This process faces no problem in case of small data, but if the amount of data increases to a huge level then more client resources are required to cache the data, which degrades the application performance, thus reducing its availability. To overcome this problem a more optimized solution is needed for paging query process. Since resources at database server are sufficient to process the query than the client server, we have used the key technologies in our proposed model to improve the paging query efficiency e.g. tuning SGA, tuning PGA, utilizing indexes, reduce swapping and query optimization.

Another major bottleneck of most of the large databases which affect paging query, is the volume of data returned to the database server from storage i.e., the time it takes to transfer the huge amount of data between the disks/storage systems and the database servers or in other words when there is full table scan involved. The smart scan model has been presented which is used to solve the problem of this unnecessary time spent moving the unrelated data from the storage tier to the database tier. This concept has three leading goals: to reduce the data volume being transferred between the database and storage tiers, to reduce CPU cycles and to reduce the disks access or I/Os.

\section{Conclusion}

In this research paper all the tools used for decision making in term of Business Intelligence explained there importance. The research explained the large amount of data used by different tools for data integration and analysis purpose for decision making by the organization. The paper mainly focused on the importance of BI system and the architecture of Online Analytical Processing (OLAP) with respect to decision support application. It explained that OLAP system provides analyst and manager the platform to improve the performance of company. Multidimensional database can be multidimensional matrices. The research is very important in term of OLAP architecture and can be further enhanced Focusing on how enterprise executives take decisions on the basis of data provided by the different sources like enterprises. In BI lots of tools have been developed to support the enterprise manager in decision making. But the tools are designed according to the requirements and have some limitation. Some tools have been developed for small scale business and some are developed for large scale business like telecom subscribers. Some tools have less data analysis capabilities and reporting limitation. But in this paper efficient data mining tools, BI framework and computation intelligence techniques have been introduced for the large scale organizations. Business Intelligence is used in an organization to analyze the data and then on the basis of this data decision are made. It results in examining their business to implement BI tools. But there are still some organizations that face challenges to be part of BI and some have implemented BI application but they are unable to get 
maximum benefits from BI because they are not fully familiar the concepts of BI and are not fully ready for BI. Enterprise managers take important decision on the basis of data provided by BI tools so nowadays no one can ignore the importance of $\mathrm{BI}$ and BI tools in the market. Business Intelligence technologies provide the activities of decision support system. Business intelligence is the IT system made for executives with knowledge and examples, concepts, and technologies. The given tools used for BI gives way to innovation or create a niche. A new Hybrid system is suggested which will be built by getting data across different sources and then combine and organize it in a centralized database. After that transformation process will be applied to it to refine and make it a base for reporting system. The different models have been studied and then the information provided by them collected and tried to find out a system which can generate a well-defined support system to assist higher executives and companies owners to take any decision for that they can increase the profit and revenue of their respective organization. In this way the proposed model will be used to build another database which would collect data from different data sources and deposit into a centralized location. For future work, a new Decision Support System would be developed with the combination of database as well as Java Application and it would be deployed practically to a wellknown business enterprise.

\section{References}

[1] V. Ciobanu, F. Pop, D. Popescu and V. Cristea, "A Distributed Approach to Business Intelligence Systems Synchronization", in Symbolic and Numeric Algorithms for Scientific Computing (SYNASC)", 2010 12th International Symposium on IEEE, (2010) September, pp. 581-585.

[2] H. You, "A Knowledge Management Approach for Real-Time Business Intelligence", in Intelligent Systems and Applications (ISA), 2010 2nd International Workshop on IEEE, (2010) May, pp. 1-4.

[3] Z. Ren, "Building Business Intelligence Application with SAP BI", in Management and Service Science, 2009. MASS'09. International Conference on IEEE, (2009) September, pp. 1-4.

[4] H. Al-Aqrabi, L. Liu, R. Hill, Z. Ding and N. Antonopoulos, "Business Intelligence Security on the Clouds: Challenges, Solutions and Future Directions”, in SOSE, (2013), pp. 137-144.

[5] F. Wang, A. Yan and L. Yang, "Combined application of cloud computation technology and Business Intelligence", in Electrical and Control Engineering (ICECE), 2011 International Conference on IEEE, (2011) September, pp. 2933-2936.

[6] U. Dayal, M. Castellanos, A. Simitsis and K. Wilkinson, "Data integration flows for business intelligence", in Proceedings of the 12th International Conference on Extending Database Technology: Advances in Database Technology, ACM, (2009) March, pp. 1-11.

[7] A. Cvitas, "Information extraction in business intelligence systems", in MIPRO, 2010 Proceedings of the 33rd International Convention, IEEE, (2010) May, pp. 1278-1282.

[8] S. Mathrani and A. Mathrani, "Leveraging Business Intelligence to Build Meta-knowledge", in System Sciences (HICSS), 2013 46th Hawaii International Conference on IEEE, (2013) January, pp. 3787-3796.

[9] S. Iqbal and M. N. A. Khan, "Yet another Set of Requirement Metrics for Software Projects", International Journal of Software Engineering \& Its Applications, vol. 6, no. 1, (2012).

[10] M. Faizan, S. Ulhaq and M. N. A. Khan, "Defect Prevention and Process Improvement Methodology for Outsourced Software Projects", Middle-East Journal of Scientific Research, vol. 19, no. 5, (2014), pp. 674-682.

[11] M. Faizan, M. N.A. Khan and S. Ulhaq, "Contemporary Trends in Defect Prevention", A Survey Report. International Journal of Modern Education \& Computer Science, vol. 4, no. 3, (2012).

[12] K. Khan, A. Khan, M. Aamir and M. N. A. Khan, "Quality Assurance Assessment in Global Software Development”, World Applied Sciences Journal, vol. 24, no. 11, (2013).

[13] M. Amir, K. Khan, A. Khan and M. N. A. Khan, "An Appraisal of Agile Software Development Process", International Journal of Advanced Science \& Technology, vol. 58, (2013).

[14] M. Umar and M. N. A. Khan, "A Framework to Separate NonFunctional Requirements for System Maintainability", Kuwait Journal of Science \& Engineering, vol. 39, no. 1 B, (2012), pp. 211- 231.

[15] M. Umar and M. N. A. Khan, "Analyzing Non-Functional Requirements (NFRs) for software development”, In IEEE 2nd International Conference on Software Engineering and Service Science (ICSESS), (2011), pp. 675-678.

[16] M. N. A. Khan and S. Ullah, "A log aggregation forensic analysis framework for cloud computing environments", Computer Fraud \& Security, vol. 2017, no. 7, (2017), pp. 11-16.

[17] M. N. A. Khan and I. Wakeman, "Machine learning for post-event timeline reconstruction", in First Conference on Advances in Computer Security and Forensics Liverpool, UK, (2006), pp. 112-121. 
[18] S. Rahman and M. N. A. Khan, "Review of live forensic analysis techniques", International Journal of Hybrid Information Technology, vol. 8, no. 2, (2015), pp. 379-88.

[19] M. N. A. Khan, C. R. Chatwin and R. C. Young, "Extracting Evidence from Filesystem Activity using Bayesian Networks", International Journal of Forensic Computer Science, vol. 1, (2007), pp. 50-63.

[20] M. N. A. Khan, "Performance analysis of Bayesian networks and neural networks in classification of file system activities", Computers \& Security, vol. 31, no. 4, (2012), pp. 391-401.

[21] M. N. A. Khan, C. R. Chatwin and R. C. Young, "A framework for post-event timeline reconstruction using neural networks", Digital Investigation, vol. 4, no. 3-4, (2007), pp. 146-157.

[22] M. S. Bashir and M. N. A. Khan, "Triage in live digital forensic analysis", International journal of Forensic Computer Science, vol. 1, (2013), pp. 35-44.

[23] M. Rafique and M. N. A. Khan, "Exploring static and live digital forensics: Methods, practices and tools", International Journal of Scientific \& Engineering Research, vol. 4, no. 10, (2013), pp. 1048-1056.

[24] R. Shehzad and M. N. A. Khan, "Integrating knowledge management with business intelligence processes for enhanced organizational learning", International Journal of Software Engineering and Its Applications, vol. 7, no. 2, (2013), pp. 83-91.

[25] M. Khalid, S. ul Haq and M. N. A. Khan, "An assessment of extreme programming based requirement engineering process", International Journal of Modern Education and Computer Science, vol. 5, no. 2, (2013), pp. 41. 